\title{
Pion to photon transition form factors with basis light-front quantization
}

\author{
Chandan Mondal $\odot,{ }^{1,2, *}$ Sreeraj Nair $\odot,{ }^{1,2, \dagger}$ Shaoyang Jia $\odot,{ }^{3,4, *}$ Xingbo Zhao ${ }^{1,2, \S}$ and James P. Vary $\odot^{3, \|}$ \\ (BLFQ Collaboration) \\ ${ }^{1}$ Institute of Modern Physics, Chinese Academy of Sciences, Lanzhou 730000, China \\ ${ }^{2}$ School of Nuclear Science and Technology, University of Chinese Academy of Sciences, \\ Beijing 100049, China \\ ${ }^{3}$ Department of Physics and Astronomy, Iowa State University, Ames, Iowa 50011, USA \\ ${ }^{4}$ Physics Division, Argonne National Laboratory, Argonne, Illinois 60439, USA
}

(Received 7 September 2021; accepted 5 November 2021; published 22 November 2021)

\begin{abstract}
We obtain the distribution amplitude (DA) of the pion from its light-front wave functions in the basis light-front quantization framework. This light-front wave function of the pion is given by the lowest eigenvector of a light-front effective Hamiltonian consisting a three-dimensional confinement potential and the color-singlet Nambu-Jona-Lasinion interaction both between the constituent quark and antiquark. The quantum chromodynamics evolution of the DA is subsequently given by the perturbative EfremovRadyushkin-Brodsky-Lepage evolution equation. Based on this DA, we then evaluate the singly and doubly virtual transition form factors in the spacelike region for $\pi^{0} \rightarrow \gamma^{*} \gamma$ and $\pi^{0} \rightarrow \gamma^{*} \gamma^{*}$ processes using the hard-scattering formalism. Our prediction for the pion-photon transition form factor agrees well with data reported by the Belle Collaboration. However, in the large $Q^{2}$ region it deviates from the rapid growth reported by the BABAR Collaboration. Meanwhile, our result on the $\pi^{0} \rightarrow \gamma^{*} \gamma^{*}$ transition form factor is also consistent with other theoretical approaches and agrees with the scaling behavior predicted by perturbative quantum chromodynamics.
\end{abstract}

DOI: 10.1103/PhysRevD.104.094034

\section{INTRODUCTION}

The parton distribution amplitudes (DAs) that play essential roles in describing the various hard exclusive processes of quantum chromodynamics (QCD) bound states [1-3] via the factorization theorem [4] are among the most basic structure functions. The DAs are therefore complementary to the parton distribution functions (PDFs) associated with inclusive processes [5-8]. Since the DAs are longitudinal projections of the hadronic wave functions obtained by integrating out the transverse momenta of the partons [1,2,9], they carry information on QCD bound states at the amplitude level. Specifically, the lowest moments of the DAs for a quark and an antiquark inside

\footnotetext{
*mondal@impcas.ac.cn

†reeraj@impcas.ac.cn

*syjia@anl.gov

§bzhao@impcas.ac.cn

"jvary@iastate.edu
}

Published by the American Physical Society under the terms of the Creative Commons Attribution 4.0 International license. Further distribution of this work must maintain attribution to the author(s) and the published article's title, journal citation, and DOI. Funded by SCOAP ${ }^{3}$. a meson are closely related to decay constants and transition form factors (TFFs) [10-13].

The meson-photon transitions with one or two virtual photons are the simplest decay processes in QCD, reflecting the structure of the meson. The associated TFFs are crucial for determining important observables, for example the hadronic light-by-light contribution to the Standard Model prediction of the muon anomalous magnetic moment [14-20], and the rates of rare pseudoscalar $(P)$ decays: $P \rightarrow$ $\overline{l l}(l \equiv e, \mu)[21,22]$. The single-virtual TFFs in the spacelike regions up to a large momentum transfer $\left(Q^{2} \sim 40 \mathrm{GeV}^{2}\right)$ have been measured experimentally by several collaborations [23-28]. The results from the BABAR Collaboration [27] demonstrate a rapid growth of $Q^{2} F_{\pi \gamma}\left(Q^{2}\right)$ in the large $Q^{2}$ region, $\left[F_{P_{\gamma}}\left(Q^{2}\right)\right.$ being the meson to photon TFF]. However, the measurement by the Belle Collaboration [26] shows that $Q^{2} F_{\pi \gamma}\left(Q^{2}\right) \rightarrow$ constant for $Q^{2}>15 \mathrm{GeV}^{2}$. The data from the Belle Collaboration are consistent with the prominent features of perturbative QCD (PQCD) $[1,29]$, where TFFs are expected to follow the asymptotic behavior of, $Q^{2} F_{P \gamma}\left(Q^{2}\right) \rightarrow$ constant as $Q^{2} \rightarrow \infty$. Unlike their results showing growth in $Q^{2} F_{\pi \gamma}\left(Q^{2}\right)$, the $B A B A R$ data [28] for the $Q^{2} F_{\left(\eta, \eta^{\prime}\right) \gamma}\left(Q^{2}\right)$ appear consistent with the PQCD 
predication. The inconsistency between the Belle and the $B A B A R$ data for $Q^{2} F_{\pi \gamma}\left(Q^{2}\right)$ and the disparities in the behaviors of $Q^{2} F_{\pi \gamma}\left(Q^{2}\right)$ and $Q^{2} F_{\left(\eta, \eta^{\prime}\right) \gamma}\left(Q^{2}\right)$ at the high $Q^{2}$ regime as reported by the $B A B A R$ Collaboration have led to various theoretical studies $[10,16,30-50]$.

While the measurements of the single-virtual TFFs having been carried out by several collaborations, information is less available on the double-virtual TFFs. Recently, the BABAR Collaboration [51] has measured for the first time the double-virtual $\gamma^{*}\left(q_{1}\right) \gamma^{*}\left(q_{2}\right) \rightarrow \eta^{\prime}$ TFF, $F_{\eta^{\prime} \gamma^{*}}\left(Q_{1}^{1}, Q_{2}^{2}\right)$, in the spacelike region where $Q_{1(2)}^{2}=$ $-q_{1(2)}^{2}>0$. Meanwhile, the meson $(M)$ TFFs for the doubly virtual $M \rightarrow \gamma^{*} \gamma^{*}$ transitions have been studied within the Dyson-Schwinger and Bethe-Salpeter framework [52], the chiral perturbation theory [53], a light-front quark model [54], the anti-de Sitter (AdS)/QCD [55,56], and the lattice QCD [16,57-60].

Our theoretical framework for meson structures is based on basis light front quantization (BLFQ), which provides an approach for solving relativistic many-body bound state structure in quantum field theories [61-77]. In this work, we evaluate the DA of the pion using the light-front wave functions (LFWFs) based on BLFQ [61], within the valence Fock sector of the pion. We then evaluate the singly and doubly virtual TFFs in the spacelike region for $\pi^{0} \rightarrow \gamma^{*} \gamma$ and $\pi^{0} \rightarrow \gamma^{*} \gamma^{*}$ transitions following the hardscattering formalism. The effective Hamiltonian includes a three-dimensional confining potential consisting of the light-front holography in the transverse direction [78], a longitudinal confinement $[65,66]$, and the color-singlet Nambu-Jona-Lasinio (NJL) interactions $[79,80]$. The colorsinglet NJL interactions account for the dynamical chiral symmetry breaking of QCD. The nonperturbative solutions for the LFWFs given by the recent BLFQ study of light mesons [67] have been applied successfully to predict the electromagnetic form factors and associated charge radii, PDFs, structure functions and generalized parton distributions of the pion [67-70]. Here, we extend our investigations of the pion to compute its singly and doubly virtual photon TFFs.

\section{BLFQ-NJL MODEL FOR THE LIGHT MESONS}

The structures of the bound states are embedded in the LFWFs obtainable as the solutions of the eigenvalue equation of the Hamiltonian $H_{\text {eff }}|\Psi\rangle=M^{2}|\Psi\rangle$, where $H_{\text {eff }}$ is the effective Hamiltonian, with $M^{2}$ being the mass squared eigenvalue of the state $|\Psi\rangle$. Within the current modeling of the meson structure through the framework of BLFQ [61], we consider an effective light-front Hamiltonian and solve for its mass eigenvalues and eigenstates at the model scale suitable for low-resolution probes. In the valence Fock sector, the effective Hamiltonian for the light mesons from Ref. [67] is given by

$$
\begin{aligned}
H_{\mathrm{eff}}= & \frac{\vec{k}_{\perp}^{2}+m_{q}^{2}}{x}+\frac{\vec{k}_{\perp}^{2}+m_{\bar{q}}^{2}}{1-x}+\kappa^{4} \vec{\zeta}_{\perp}^{2} \\
& -\frac{\kappa^{4}}{\left(m_{q}+m_{\bar{q}}\right)^{2}} \partial_{x}\left(x(1-x) \partial_{x}\right)+H_{\mathrm{NJL}}^{\mathrm{eff}},
\end{aligned}
$$

where $m_{q}\left(m_{\bar{q}}\right)$ is the mass of the quark (antiquark), and $\vec{k}_{\perp}$ is the relative transverse momentum. The parameter $\kappa$ represents the strength of the confinement. The transverse confinement is adopted from the light-front holography, where the holographic variable is defined as $\vec{\zeta}_{\perp} \equiv$ $\sqrt{x(1-x)} \vec{r}_{\perp}$ [78]. The variable $\vec{r}_{\perp}$ is conjugated to $\vec{k}_{\perp}$ and measures the transverse separation between the quark and antiquark. The $x$ derivative in the longitudinal confining potential [65] is defined as $\partial_{x} f\left(x, \vec{\zeta}_{\perp}\right)=\partial f\left(x, \vec{\zeta}_{\perp}\right) /\left.\partial x\right|_{\vec{\zeta}_{\perp}}$. The effective Hamiltonian also includes $H_{\mathrm{NJL}}^{\text {eff }}$ that corresponds to the color-singlet NJL interaction to account for the chiral dynamics [79]. For the positively charged pion, the NJL interaction is given by [67]

$$
\begin{aligned}
H_{\mathrm{NJL}, \pi}^{\mathrm{eff}}= & G_{\pi}\left\{\bar{u}_{\mathrm{us} 1^{\prime}}\left(p_{1}^{\prime}\right) u_{\mathrm{us} 1}\left(p_{1}\right) \bar{v}_{\mathrm{d} s 2}\left(p_{2}\right) v_{\mathrm{d} s 2^{\prime}}\left(p_{2}^{\prime}\right)\right. \\
& +\bar{u}_{\mathrm{us} 1^{\prime}}\left(p_{1}^{\prime}\right) \gamma_{5} u_{\mathrm{us} 1}\left(p_{1}\right) \bar{v}_{\mathrm{d} s 2}\left(p_{2}\right) \gamma_{5} v_{\mathrm{ds} 2^{\prime}}\left(p_{2}^{\prime}\right) \\
& \left.+2 \bar{u}_{\mathrm{us} 1^{\prime}}\left(p_{1}^{\prime}\right) \gamma_{5} v_{\mathrm{d} s 2^{\prime}}\left(p_{2}^{\prime}\right) \bar{v}_{\mathrm{d} s 2}\left(p_{2}\right) \gamma_{5} u_{\mathrm{us} 1}\left(p_{1}\right)\right\},
\end{aligned}
$$

which is obtained from the NJL Lagrangian in the two flavor NJL model after the Legendre transform [79,81-83]. The nonitalic and italic subscripts in the Dirac spinors, $u_{\mathrm{f} s}(p)$ and $v_{\mathrm{fs}}(p)$, respectively, represent the flavors and the spins. Meanwhile, $p_{1}$ and $p_{2}$ are the momenta of the valence quark and the valence antiquark, respectively. The coefficient $G_{\pi}$ is the coupling constant of the NJL interaction. In this interaction, only the combinations of Dirac bilinears relevant to the valence Fock sector LFWFs of the pion have been included with the instantaneous terms due to the NJL interactions neglected. The explicit expressions for the matrix elements of the NJL interactions within the BLFQ framework can be found in Ref. [67].

To compute the Hamiltonian matrix we follow BLFQ [61] and adopt the two-dimensional (2D) harmonic oscillator basis functions, to describe the transverse degrees of freedom, which are defined as [65]

$$
\begin{aligned}
\phi_{n m}\left(\vec{q}_{\perp} ; \kappa\right)= & \frac{1}{\kappa} \sqrt{\frac{4 \pi n !}{(n+|m|) !}}\left(\frac{\left|\vec{q}_{\perp}\right|}{\kappa}\right)^{|m|} \\
& \times \exp \left(-\frac{\vec{q}_{\perp}^{2}}{2 \kappa^{2}}\right) L_{n}^{|m|}\left(\frac{\vec{q}_{\perp}^{2}}{\kappa^{2}}\right) e^{i m \varphi},
\end{aligned}
$$

with $\tan (\varphi)=q_{2} / q_{1}, L_{n}^{|m|}(z)$ is the associated Laguerre polynomial, $n$ and $m$ are the radial and the angular quantum numbers, respectively. On the other hand, the basis functions in the longitudinal direction are defined as [65] 


$$
\chi_{l}(x ; \alpha, \beta)=\sqrt{4 \pi(2 l+\alpha+\beta+1)} \sqrt{\frac{\Gamma(l+1) \Gamma(l+\alpha+\beta+1)}{\Gamma(l+\alpha+1) \Gamma(l+\beta+1)}} x^{\beta / 2}(1-x)^{\alpha / 2} P_{l}^{(\alpha, \beta)}(2 x-1),
$$

where $P_{l}^{(\alpha, \beta)}(z)$ is the Jacobi polynomial with the dimensionless parameters $\alpha=2 m_{\bar{q}}\left(m_{q}+m_{\bar{q}}\right) / \kappa^{2}$, $\beta=2 m_{q}\left(m_{q}+m_{\bar{q}}\right) / \kappa^{2}$, and $l=0,1,2, \ldots$ Using basis functions specified in Eqs. (3) and (4), the valence wave function in a given spin combination is expanded as

$$
\psi_{r s}\left(x, \vec{k}_{\perp}\right)=\sum_{n, m, l}\langle n, m, l, r, s \mid \psi\rangle \phi_{n m}\left(\frac{\vec{k}_{\perp}}{\sqrt{x(1-x)}} ; \kappa\right) \chi_{l}(x ; \alpha, \beta),
$$

where the coefficients $\langle n, m, l, r, s \mid \psi\rangle$ represent the LFWFs in our BLFQ basis representation obtained from diagonalizing the truncated Hamiltonian. We truncate the infinite dimensional Hilbert space of the valence Fock sector to a finite dimension by imposing the following restrictions on the quantum numbers [67]:

$0 \leq n \leq N_{\max }, \quad-2 \leq m \leq 2, \quad 0 \leq l \leq L_{\max }$,

where $L_{\max }$ specifies the basis resolution in the longitudinal direction, while $N_{\max }$ controls the transverse momentum covered by $2 \mathrm{D}$ harmonic oscillator functions. Since the NJL interactions do not couple to $|m| \geq 3$ basis states, we have a natural truncation for $m$ [67]. The LFWFs of the mesons $\psi_{r s}\left(x, \vec{k}_{\perp}\right)$ are normalized as

$$
\sum_{r, s} \int_{0}^{1} \frac{d x}{2 x(1-x)} \int \frac{d^{2} \vec{k}_{\perp}}{(2 \pi)^{3}}\left|\psi_{r s}\left(x, \vec{k}_{\perp}\right)\right|^{2}=1 .
$$

Parameters in the BLFQ-NJL model have been fixed to generate the ground state masses of the light pseudoscalar as well as the vector mesons and the charge radii of the $\pi^{+}$ and the $K^{+}$[67]. The LFWFs in this model provide a high quality description of the electromagnetic form factors [67], PDFs for the pion and the kaon and pion-nucleus induced Drell-Yan cross sections [68,69]. The coefficient $\langle n, m, l, r, s \mid \psi\rangle$ for the neutral pion is assumed to be identical to that of the charged pion, resulting in the only difference between their wave functions being flavor.

\section{PARTON DISTRIBUTION AMPLITUDE}

The DAs are defined using the lightlike separated gauge invariant vacuum-to-meson matrix elements. Explicitly in the light-front formalism, the leading-twist DAs $\phi_{M}(x ; \mu)$ in the light cone gauge for a pseudoscalar are defined by $[1,84]$

$$
\begin{aligned}
& \left\langle 0\left|\bar{\psi}(z) \gamma^{+} \gamma_{5} \psi(-z)\right| M(p)\right\rangle_{\mu} \\
& \quad=\left.\mathrm{i} p^{+} f_{M} \int_{0}^{1} \mathrm{~d} x e^{\mathrm{i} p^{+} z^{-}\left(x-\frac{1}{2}\right)} \phi_{M}(x ; \mu)\right|_{z^{+}, \vec{z}_{\perp}=0},
\end{aligned}
$$

where $f_{M}$ are the decay constants. The nonlocal matrix elements as well as the DAs depend on the scale $\mu$, the renormalization scale which we take to be the UV cutoff. Following these definitions, the DAs are normalized to unity:

$$
\int_{0}^{1} \mathrm{~d} x \phi(x ; \mu)=1
$$

In terms of LFWF, the DAs of pseudoscalar states can be written as [1]

$\phi\left(x, \mu_{0}\right)=\frac{2 \sqrt{2 N_{c}}}{f_{M}} \frac{1}{\sqrt{x(1-x)}} \int \frac{\mathrm{d}^{2} \vec{k}_{\perp}}{2(2 \pi)^{3}} \psi_{\uparrow \downarrow-\downarrow \uparrow}^{\lambda=0}\left(x, \vec{k}_{\perp}\right)$,

where $\psi_{\uparrow \downarrow-\downarrow \uparrow}=\left(\psi_{\uparrow \downarrow}-\psi_{\downarrow \uparrow}\right) / \sqrt{2}$ and $N_{c}$ is the number of colors. In the BLFQ-NJL model, we use $N_{c}=3$. We compute the DA of the pion at the model scale using the LFWFs given in Eq. (5). Recall that the flavor wave function of the neutral pion is the only difference compared to the wave function of the charged pion in the BLFQNJL model.

The QCD evolution of the DA is specified by the Efremov-Radyushkin-Brodsky-Lepage (ERBL) equations $[1,9,85]$. In a Gegenbauer basis, one has [86]

$$
\phi(x, \mu)=6 x(1-x) \sum_{n=0}^{\infty} C_{n}^{3 / 2}(2 x-1) a_{n}(\mu),
$$

where $C_{n}^{\frac{3}{2}}(2 x-1)$ is a Gegenbauer polynomial and

$$
\begin{aligned}
a_{n}(\mu)= & \frac{2}{3} \frac{2 n+3}{(n+1)(n+2)}\left(\frac{\alpha_{s}(\mu)}{\alpha_{s}\left(\mu_{0}\right)}\right)^{\gamma_{n}^{0} / 2 \beta_{0}} \\
& \times \int_{0}^{1} \mathrm{~d} x C_{n}^{3 / 2}(2 x-1) \phi\left(x, \mu_{0}\right),
\end{aligned}
$$

with 


$$
\begin{aligned}
\gamma_{n}^{(0)} & =-2 C_{\mathrm{F}}\left[3+\frac{2}{(n+1)(n+2)}-4 \sum_{k}^{n+1} \frac{1}{k}\right] ; \\
\beta_{0} & =\frac{11}{3} C_{\mathrm{A}}-\frac{2}{3} n_{\mathrm{f}},
\end{aligned}
$$

in the leading order (LO). Here, the color factors are given by $C_{\mathrm{F}}=\frac{4}{3}$ and $C_{\mathrm{A}}=3$. We take $n_{\mathrm{f}}=3$ to be the number of active flavors. The strong running coupling is given by

$$
\alpha_{\mathrm{s}}(\mu)=\frac{4 \pi}{\beta_{0} \ln \left(\mu^{2} / \Lambda_{\mathrm{QCD}}^{2}\right)},
$$

with $\Lambda_{\mathrm{QCD}}=0.204 \mathrm{GeV}$ being the QCD scale parameter. It is also useful to compute the moments in order to quantitatively compare with other theoretical predictions. The $p$ th moment of the DA is defined as

$$
\left\langle z_{p}\right\rangle=\int_{0}^{1} d x z^{p} \phi(x, \mu)
$$

where $z \equiv(2 x-1)$ when $p \geq 1$ and $z \equiv x$ for $p=-1$. Meanwhile, the moments of the DA are directly related to the Gegenbauer coefficients in Eq. (11). The second, fourth, and sixth moments are expressed as [44]

$$
\begin{gathered}
\left\langle z_{2}\right\rangle=\frac{12}{35} a_{2}+\frac{1}{5}, \\
\left\langle z_{4}\right\rangle=\frac{3}{35}+\frac{8}{35} a_{2}+\frac{8}{77} a_{4}, \\
\left\langle z_{6}\right\rangle=\frac{1}{21}+\frac{12}{77} a_{2}+\frac{120}{1001} a_{4}+\frac{64}{2145} a_{6} .
\end{gathered}
$$

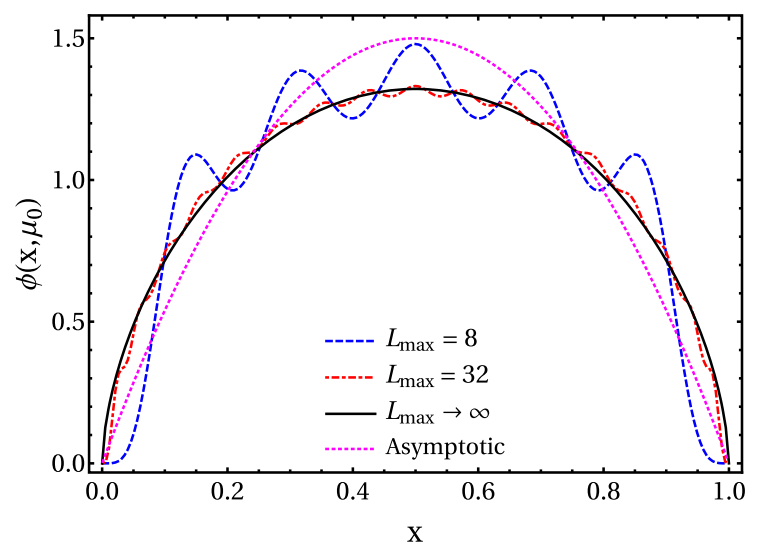

The LFWFs of the valence quarks in the light mesons have been solved in the BLFQ framework using the NJL interactions as discussed in the Sec. II. We employ the wave functions obtained with truncated basis to compute the DA of the pion using Eq. (10). On the left panel of Fig. 1, we show the valence quark DA of the pion at the model scale using the basis truncation $N_{\max }=8$ with $L_{\max }=8,32$, and extrapolation to $\infty$. The oscillations on the DA are numerical artifacts, while with increasing $L_{\max }$ the DA tends toward a smooth function with decreasing oscillation amplitude about a single-peaked function. The DA for $L_{\max } \rightarrow \infty$ is therefore fitted to the following functional form [67]:

$$
\phi\left(x, \mu_{0}\right)=\frac{x^{a}(1-x)^{b}}{B(a+1, b+1)},
$$

with $a=b=0.6$, where $B(a+1, b+1)$ is the Euler beta function. We illustrate the pion valence quark DA after QCD evolution on the right panel of Fig. 1. Explicitly, we evolve our input DA from the model scale $\mu_{0}^{2}=$ $0.120 \pm 0.012 \mathrm{GeV}^{2}$ [69] to the experimental scale of the FNAL-E-791 experiment, $10 \mathrm{GeV}^{2}$ [87]. The initial scale was determined by requiring the PDF result after QCD evolution utilizing the LO DGLAP equation to fit the pion valence quark PDF data from the FNAL-E-615 experiment [88]. As can be seen, our evolved DA is in excellent agreement with the FNAL-E-791 data. On the other hand, the pion DA in the BLFQ-NJL model is very close to the asymptotic DA already at $\mu^{2}=10 \mathrm{GeV}^{2}$.

The numerical values of the first three nonvanishing moments and the inverse moment of the pion DA in the BLFQ-NJL model are presented in Table I. We compare our predictions with the results obtained from various theoretical approaches. Our predictions for $z_{2}$ and/or $z_{4}$

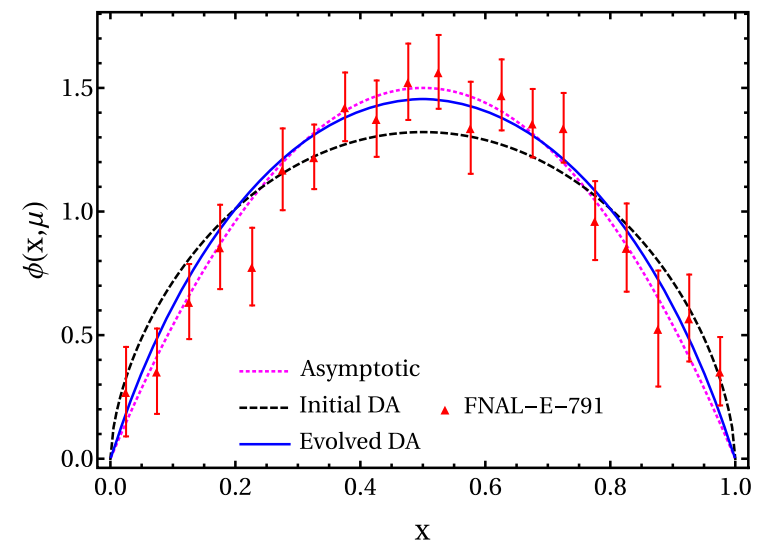

FIG. 1. The valence quark DA of the pion in the BLFQ-NJL model. Left panel: the DAs for $L_{\max }=8$ (blue dashed) $L_{\text {max }}=32$ (red dash dotted) and extrapolated to $L_{\max } \rightarrow \infty$ that fits to Eq. (19) with $a=b=0.62$ (black solid) at the model scale. Right panel: the evolved DA from the initial scale $\left(\mu_{0}^{2}=0.120 \pm 0.012 \mathrm{GeV}^{2}\right)$ using ERBL equations to the experimental scale of $10 \mathrm{GeV}^{2}$. The black dashed line corresponds to the DA at the initial scale, while the blue solid line represents the evolved DA. Our result is compared with the FNAL-E-791 data [87] and the asymptotic DA (magenta dotted): $6 x(1-x)$ [86]. 
TABLE I. Our predictions for the first three nonvanishing moments and inverse moment of the pion DA, compared to other theoretical predictions.

\begin{tabular}{|c|c|c|c|c|c|}
\hline & $\mu(\mathrm{GeV})$ & $\left\langle z_{2}\right\rangle$ & $\left\langle z_{4}\right\rangle$ & $\left\langle z_{6}\right\rangle$ & $\left\langle x^{-1}\right\rangle$ \\
\hline BLFQ-NJL (this work) & 1,2 & $0.221,0.217$ & $0.099,0.097$ & $0.057,0.055$ & $3.21,3.17$ \\
\hline Playkurtic [90] & 2 & $0.220_{-0.006}^{+0.009}$ & $0.098_{-0.005}^{+0.008}$ & $\ldots$ & $3.13_{-0.10}^{+0.14}$ \\
\hline NLC sum rules [93] & 2 & $0.248_{-0.015}^{+0.016}$ & $0.108_{-0.03}^{+0.05}$ & $\cdots$ & $3.16(9)$ \\
\hline LF quark model [44] & $\sim 1$ & $0.24(22)$ & $0.11(9)$ & $0.07(5)$ & $\ldots$ \\
\hline Sum rules $[91]$ & 1 & 0.24 & 0.11 & $\ldots$ & $\cdots$ \\
\hline AdS/QCD [55] & $\sim 1$ & 0.25 & 0.125 & 0.078 & 3.98 \\
\hline LF holographic $(B=0)[46]$ & 1,2 & $0.180,0.185$ & $0.067,0.071$ & $\cdots$ & $2.81,2.85$ \\
\hline LF holographic $(B \gg 1)[46]$ & 1,2 & $0.200,0.200$ & $0.085,0.085$ & $\cdots$ & $2.93,2.95$ \\
\hline Renormalon model [98] & 1 & 0.28 & 0.13 & $\cdots$ & $\ldots$ \\
\hline Instanton vacuum (MIA 1) [92] & 1,2 & $0.237,0.218$ & $0.112,0.094$ & $0.066,0.052$ & $\cdots$ \\
\hline Instanton vacuum (MIA 2) [92] & 1,2 & $0.239,0.220$ & $0.113,0.096$ & $0.067,0.053$ & $\cdots$ \\
\hline Sum rules $[2]$ & 2 & 0.343 & 0.181 & $\cdots$ & 4.25 \\
\hline Dyson-Schwinger [RL, DB] [99] & 2 & $0.280,0.251$ & $0.151,0.128$ & $\cdots$ & $5.5,4.6$ \\
\hline QCD background field theory sum rule [47] & 1 & $0.271(13)$ & $0.138(10)$ & $0.087(6)$ & 3.95 \\
\hline QCD background field theory sum rule [47] & 2 & $0.254(10)$ & $0.125(7)$ & $0.077(6)$ & 3.33 \\
\hline Lattice QCD [100] & 2 & $0.28(1)(2)$ & $\cdots$ & $\cdots$ & $\cdots$ \\
\hline Lattice QCD [94] & 2 & $0.2361(41)(39)$ & $\cdots$ & $\cdots$ & $\cdots$ \\
\hline Lattice QCD [101] & 2 & $0.27(4)$ & $\cdots$ & $\cdots$ & $\cdots$ \\
\hline Lattice QCD [95] & 2 & $0.2077(43)$ & $\cdots$ & $\cdots$ & $\cdots$ \\
\hline Lattice QCD [96] & 2 & $0.234(6)(6)$ & $\cdots$ & $\cdots$ & $\cdots$ \\
\hline Lattice QCD [97] & 2 & $0.244(30)$ & $\cdots$ & $\ldots$ & $\cdots$ \\
\hline Asymptotic QCD & $\infty$ & 0.200 & 0.086 & 0.048 & 3.00 \\
\hline
\end{tabular}

roughly agree with those in Refs. [44,46,89-97]. For $z_{6}$, the numerical values are compared with Refs. [44,47,55,92], with our predictions being close to the results evaluated from the QCD instanton vacuum [92]. The inverse moment of the pion DA in the BLFQ-NJL model is in good agreement with Refs. [90,93], while differing from other predictions summarized in Table I.

Based on the BLFQ-NJL model, the decay constant for the pion is given in Table II. In order to gain a clear impression on how basis truncation affects the decay constant, we include the numerical values with basis cutoffs $N_{\max }=8$ with $L_{\max }=8,16$, and 32 to demonstrate a good convergence trend. With $L_{\max }=32$ we predict $f_{\pi}=145.3 \mathrm{MeV}$, while the experimental data is $f_{\pi}^{\exp }=$ 130.2 MeV [102]. Note that the decay constants computed here differ from the results presented in Ref. [67], where an overall factor $(\sqrt{2})$ was erroneously included.

We now use the Alder-Bell-Jackiw anomaly relations $[103,104]$ to compute the pion-photon TFF at zero momentum transfer as follows $[42,46]$ :

$$
F_{\pi \gamma}^{\mathrm{ABJ}}(0)=\frac{1}{2 \sqrt{2} \pi^{2} f_{\pi^{0}}},
$$

so that we can evaluate the radiative decay width using

$$
\Gamma_{\pi \rightarrow \gamma \gamma}=\frac{\pi}{4} \alpha_{\mathrm{EM}}^{2} M_{\pi}^{3}\left|F_{\pi \gamma}(0)\right|^{2}
$$

Our results are presented in Table II, where we find reasonable agreement with the experimental value [102]. With the largest basis size in our current calculation, i.e., $\quad N_{\max }=8$ and $L_{\max }=32$, we obtain $\Gamma_{\pi \rightarrow \gamma \gamma}=$ $6.98 \times 10^{-3} \mathrm{keV}$, whereas the experimentally measured value is $\Gamma_{\pi \rightarrow \gamma \gamma}=7.82 \times 10^{-3} \mathrm{keV}$.

With the original model parameters given in Ref. [67], our prediction for the pion decay constant is somewhat larger than the experimental data, which effectively leads to a slightly smaller radiative decay width compared to the experimental value. However, if we relax the constraint from the charge radius, we can modify the model

TABLE II. Our predictions for the decay constant, $f_{\pi}$, of the pion and the radiative decay width, $\Gamma_{\pi \rightarrow 2 \gamma}$, compared to the measured values from Particle Data Group (PDG) [102]. The BLFQ-NJL model results are quoted using the LFWFs at the basis cutoff $N_{\max }=8$ and $L_{\max }=8,16$, and 32, respectively.

\begin{tabular}{lcccc}
\hline \hline$\left[N_{\max }, L_{\max }\right] \rightarrow$ & {$[8,8]$} & {$[8,16]$} & {$[8,32]$} & Experimental data [102] \\
\hline$f_{\pi}(\mathrm{MeV})$ & 142.8 & 144.8 & 145.3 & 130.2 \\
$\Gamma_{\pi \rightarrow 2 \gamma}(\mathrm{keV})$ & $7.22 \times 10^{-3}$ & $7.03 \times 10^{-3}$ & $6.98 \times 10^{-3}$ & $(7.82 \pm 0.22) \times 10^{-3}$ \\
\hline \hline
\end{tabular}


parameters into $m_{q}=356.77 \mathrm{MeV}, \kappa=182.35 \mathrm{MeV}$, and $G_{\pi}=2.6573 \times 10^{-5} \mathrm{MeV}$ for the basis cutoff $N_{\max }=8$ and $L_{\max }=32$ in order to reproduce the experimental decay constant.

\section{PION TO PHOTONS TRANSITION FORM FACTORS}

The meson-photon TFF, $F_{M \gamma}\left(Q^{2}\right)$, of a pseudoscalar meson $(M)$ for the $M \rightarrow \gamma^{*} \gamma$ decay is defined through the matrix element of electromagnetic current as [1]

$$
\left\langle\gamma(P-q)\left|J^{\mu}\right| M(P)\right\rangle=-i e^{2} F_{M \gamma}\left(Q^{2}\right) \epsilon^{\mu \nu \rho \sigma} P_{\nu} \epsilon_{\rho} q_{\sigma},
$$

where $P$ and $q$ are the momenta of the meson and the virtual photon, respectively. The invariant 4-momentum transfer squared $Q^{2}=-q^{2}>0$ and $\epsilon_{\rho}$ is the transverse polarization vector of the final (on shell) photon. The TFF, $F_{M \gamma}\left(Q^{2}\right)$, can be evaluated from the convolution of a hard scattering amplitude (HSA), $T_{\mathrm{H}}\left(x, Q^{2}\right)$, calculable in perturbation theory, with a nonperturbative DA $[1,55,105]$ using

$Q^{2} F_{\pi \gamma}\left(Q^{2}\right)=\frac{\sqrt{2}}{3} f_{\pi} \int_{0}^{1} \mathrm{~d} x T_{\mathrm{H}}\left(x, Q^{2}\right) \phi(x,(1-x) Q)$,

where $T_{\mathrm{H}}\left(x, Q^{2}\right)$ to the next-to-leading order (NLO) is given by [29,106-109],

$$
\begin{aligned}
T_{\mathrm{H}}\left(x, Q^{2}\right)= & \frac{1}{1-x}+\frac{\alpha_{s}\left(\mu_{\mathrm{R}}\right)}{4 \pi} C_{\mathrm{F}} \frac{1}{1-x}\left[-9-\frac{1-x}{x} \ln (1-x)\right. \\
& \left.+\ln ^{2}(1-x)+\{3+2 \ln (1-x)\} \ln \left(\frac{Q^{2}}{\mu_{\mathrm{R}}^{2}}\right)\right] .
\end{aligned}
$$

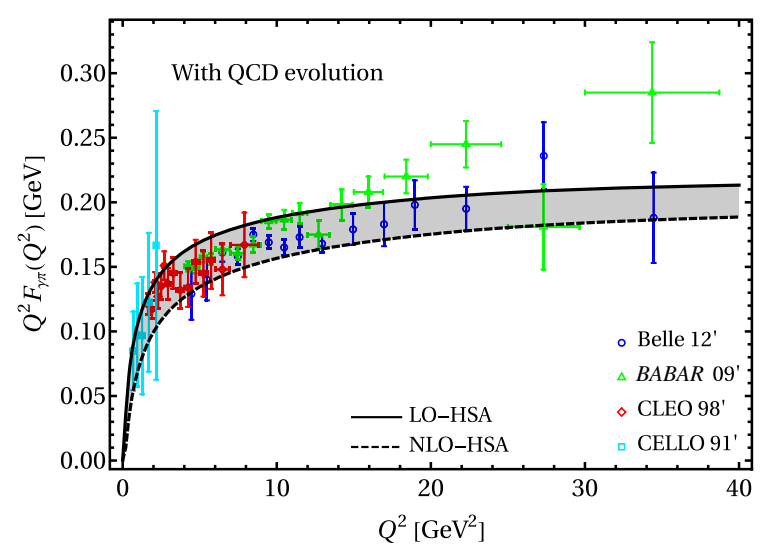

For simplicity, the regularization scale, $\mu_{\mathrm{R}}^{2}$, is taken as $\mu_{\mathrm{R}}^{2}=Q^{2}$ to eliminate large logarithm terms. At leading order, only the first term in Eq. (24) contributes to the TFF. Using the standard hard scattering approach, the NLO corrections have been studied in Refs. [29,106-109] under the assumption that $\phi(x,(1-x) Q) \simeq \phi(x, Q)$. The replacement is reasonable for the behavior at the asymptotic limit $Q^{2} \rightarrow \infty$. However, this approximation is not well justified for the calculation below the asymptotic region, where one needs to take into account the ERBL evolution effects. A proper treatment of the NLO calculations with the evolution effects and $\phi(x,(1-x) Q)$ has been illustrated in Ref. [55].

Inserting the evolved DA from Eq. (11) into Eq. (23) we evaluate $Q^{2} F_{\pi \gamma}\left(Q^{2}\right)$ with the ERBL evolution considered. Figure 2 (left panel) shows our results for the pion to photon TFF, $Q^{2} F_{\pi \gamma}\left(Q^{2}\right)$, in the BLFQ-NJL model and compares with the available experimental data from the Belle [26], the BABAR [27], the CLEO [110], and the CELLO [111] Collaborations. The gray band corresponds to the correction in the TFF due to the $\alpha_{s}$ order correction in the HSA in Eq. (24). We need to set $(1-x) Q=\mu_{0}$ for $(1-x) Q<\mu_{0}$ to ensure the convergence of the integration in Eq. (23). We obtain the TFF results with the BLFQ basis truncation: $N_{\max }=8$ with $L_{\max }=32$ and find good agreement of our calculated pion TFF with the experiments performed by the Belle [26], the CLEO [110], and the CELLO [111] Collaborations. However, it deviates from the rapid growth in the large $Q^{2}$ region reported by the $B A B A R$ Collaboration [27]. There are also theoretical studies suggesting that the BABAR data are incompatible with QCD calculations [36,112-114]. It has also been demonstrated in Ref. [55] that the explanation of $B A B A R$ data at large $Q^{2}$ with the QCD calculations using the asymptotic QCD, AdS/QCD, and Chernyak-Zhitnitsky models for the pion DA is not possible, but can be

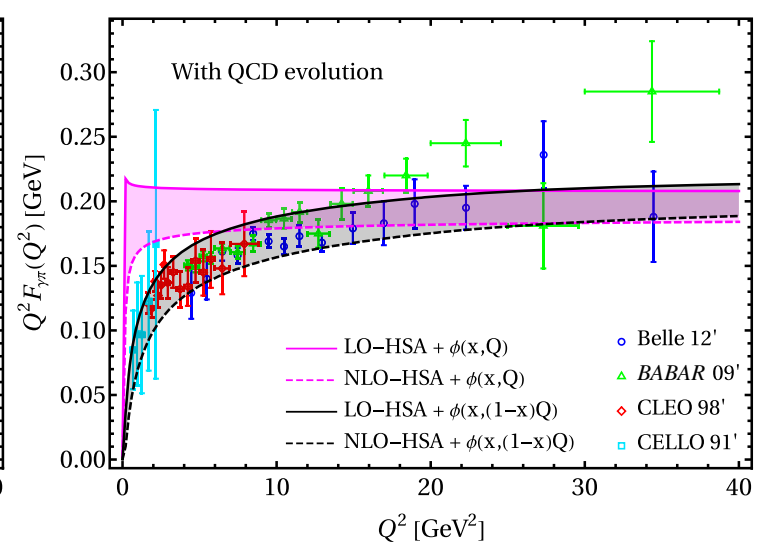

FIG. 2. The $\pi^{0} \rightarrow \gamma^{*} \gamma$ transition form factor with BLFQ basis truncation $N_{\max }=8$ and $L_{\max }=32$. Left panel: the solid and dashed lines correspond to the results with LO and NLO hard scattering amplitudes, respectively. Right panel: comparison of the TFFs evaluated with the DAs $\phi(x, Q)$ (magenta lines) and $\phi(x,(1-x) Q)$ (black lines) in Eq. (23). Effect of the scale evolution is considered following Eq. (11). 


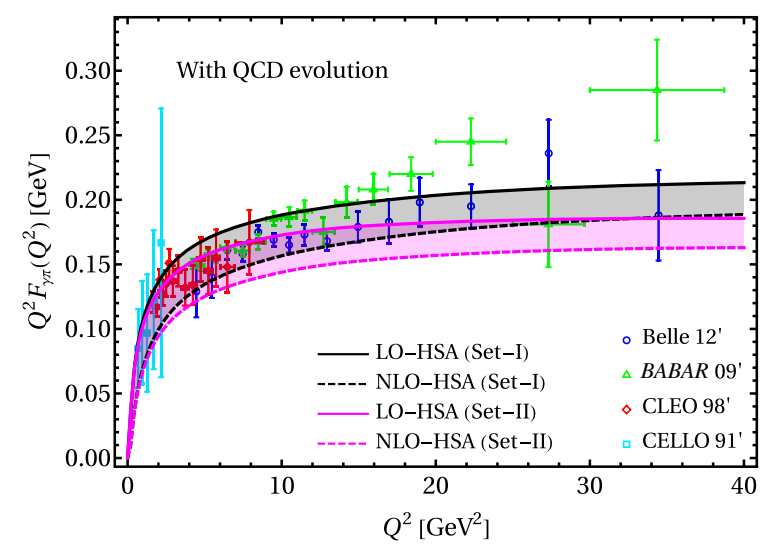

FIG. 3. Comparison of the $\pi^{0} \rightarrow \gamma^{*} \gamma$ transition form factors evaluated with the original model parameters (Set-I) given in Ref. [67] (black lines) and the readjusted parameters (Set-II) that fit the pion experimental pion decay constant (magenta lines) both with BLFQ basis truncation $N_{\max }=8$ and $L_{\max }=32$.

accommodated by a flat modeling of the pion DA [10,55]. However, the calculations with such a DA underestimate significantly the pion TFF at low $Q^{2}$. Such a DA also shows a trend that would appear to violate the Brodsky-Lepage limit of $Q^{2} F_{\pi \gamma}\left(Q^{2} \rightarrow \infty\right)=2 f_{\pi}$ [55]. Except those using the flat modeling of the pion DA $[10,31,115,116]$, there exist phenomenological studies reproducing the BABAR data for the pion to photon TFF [33,34,117-122].

In Fig. 2 (right panel), we compare the results calculated using $\phi(x,(1-x) Q)$ and $\phi(x, Q)$ in Eq. (23). We notice that it is a reasonable approximation to use $\phi(x, Q)$ in the perturbative regime, where both $\phi(x,(1-x) Q)$ and $\phi(x, Q)$ lead to almost identical pion TFF. However, using $\phi(x, Q)$ fails to reproduce the experimental data at low $Q^{2}$ region, particularly for the $Q^{2}<10 \mathrm{GeV}^{2}$, whereas the results using $\phi(x,(1-x) Q)$ agree well with the available data.

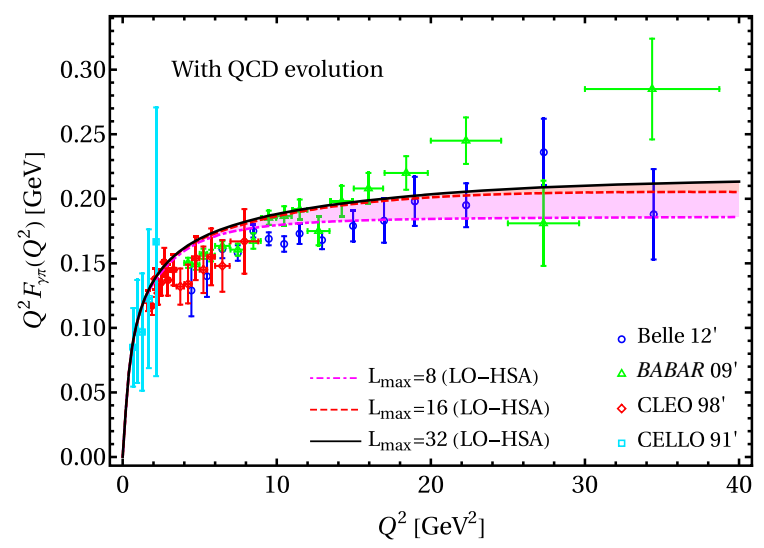

Parameters in the BLFQ-NJL model have been fixed to fit the charge radius of the pion [67], while they lead to a slightly larger pion decay constant compared to the experimental data as summarized in Table II. However, the experimental the decay constant can be obtained by adjusting the model parameters into those mentioned by the end of Sec. III. In order to gain a clear impression on how the change in parameters affects $Q^{2} F_{\pi \gamma}\left(Q^{2}\right)$, in Fig. 3 we present a comparison of the singly virtual pion to two photon TFF computed using the original model's parameters given in Ref. [67] and the modified parameters. We find that the two sets of parameters provide qualitatively similar results for this TFF at small $Q^{2}$. However, $F_{\pi \gamma}\left(Q^{2}\right)$ with new parameters is narrower than that obtained using the original model's parameters, which accounts for the difference in the TFF for large $Q^{2}$.

The sensitivity of the BLFQ-NJL model prediction to basis truncation is shown in Fig. 4, where we present the results for $\left\{N_{\max }, L_{\max }\right\} \equiv\{8,8\},\{8,16\}$, and $\{8,32\}$. The results show a good convergence trend over the range of $Q^{2}$ as evident by finding that the $L_{\max }=16$ and $L_{\max }=32$ results nearly coincide with each other in contrast to the $L_{\max }=8$ results presented in Fig. 4 . This observed convergence in the TFFs is reassuring since the DAs are also reasonably well converged as can be seen in the left panel of Fig. 1. The difference between the $L_{\max }=32$ and 8 values is presented as our uncertainty estimate in the pion TFF.

We now turn our attention to the case in which the photons as decay products are both off mass shell, i.e., for the TFF $F_{\pi \gamma^{*}}\left(Q_{1}^{2}, Q_{2}^{2}\right)$. This pion to doubly virtual photons $\left(\pi^{0} \rightarrow \gamma^{*} \gamma^{*}\right)$ TFF can be obtained by replacing the hardscattering amplitude $T_{\mathrm{H}}$ in the previous analysis with an appropriate expression. The TFF, $F_{M \gamma^{*}}\left(Q_{1}^{2}, Q_{2}^{2}\right)$, can be expressed from the convolution of a $\operatorname{HSA}, T_{\mathrm{H}}\left(x, Q_{1}^{2}, Q_{2}^{2}\right)$ with a nonperturbative DA $[1,55,105]$,

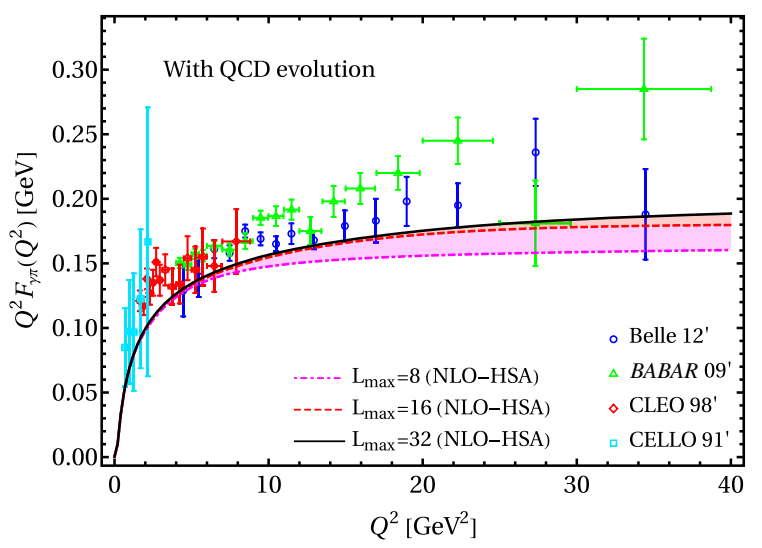

FIG. 4. Sensitivity of the $\pi^{0} \rightarrow \gamma^{*} \gamma$ transition form factor to BLFQ basis truncation. The magenta dot-dashed, red dashed, and black solid lines correspond to the $\left\{N_{\max }, L_{\max }\right\} \equiv\{8,8\},\{8,16\}$, and $\{8,32\}$, respectively. Left and right panels represent the results with LO and NLO hard scattering amplitude, respectively. 
TABLE III. The transition form factors $F_{\pi \gamma^{*}}\left(Q_{1}^{2}, Q_{2}^{2}\right)$ (in units of $\left.10^{-3} \mathrm{GeV}^{-1}\right)$ for some $\left(Q_{1}^{2}, Q_{2}^{2}\right)$ values (in units of $\mathrm{GeV}^{2}$ ) compared with the LFQM [54], the vector meson dominance (VMD) model [54], and the PQCD predictions. The first and second values for each row entry in the second and third columns correspond to the basis truncations $L_{\max }=8$ and 32, respectively. The difference between the $L_{\max }=8$ and 32 values are presented as our uncertainty estimate.

\begin{tabular}{lccccrr}
\hline \hline & $F_{\pi \gamma^{*}}^{\mathrm{LO}}$ & $F_{\pi \gamma^{*}}^{\mathrm{NLO}}$ & PQCD & PQCD & & \\
$\left(Q_{1}^{2}, Q_{2}^{2}\right)$ & (this work) & (this work) & LO & NLO & LFQM [54] & VMD [54] \\
\hline$(6.48,6.48)$ & $10.39-10.56$ & $9.59-9.75$ & 9.52 & 8.78 & 9.08 & $1.957 \pm 0.022$ \\
$(16.85,16.85)$ & $3.99-4.06$ & $3.73-3.79$ & 3.66 & 2.69 & 3.58 & $0.322 \pm 0.004$ \\
$(14.83,4.27)$ & $7.55-7.72$ & $7.00-7.14$ & 6.91 & 6.39 & 6.76 & $1.301 \pm 0.014$ \\
$(38.11,14.95)$ & $2.65-2.69$ & $2.48-2.52$ & 2.42 & 2.27 & 2.40 & $0.163 \pm 0.002$ \\
$(45.63,45.63)$ & $1.47-1.50$ & $1.39-1.41$ & 1.35 & 1.33 & 1.33 & $0.046 \pm 0.001$ \\
\hline \hline
\end{tabular}

$F_{\pi \gamma^{*}}\left(Q_{1}^{2}, Q_{2}^{2}\right)=\frac{\sqrt{2}}{3} f_{\pi} \int_{0}^{1} \mathrm{~d} x T_{\mathrm{H}}^{\gamma^{*} \gamma^{*} \rightarrow \pi^{0}}\left(x, Q_{1}^{2}, Q_{2}^{2}\right) \phi(x, \bar{Q})$,

where we assume $\bar{Q}=(1-x) Q_{1}+x Q_{2}$ and at the leading order $T_{\mathrm{H}}$ has the form

$$
T_{\mathrm{H}}^{\gamma^{*} \gamma^{*} \rightarrow \pi^{0}}\left(x, Q_{1}^{2}, Q_{2}^{2}\right)=\frac{1}{(1-x) Q_{1}^{2}+x Q_{2}^{2}} .
$$

Note that the singly virtual TFF $F_{\pi \gamma}\left(Q^{2}\right)$ can be obtained by setting one of the momentum transfers to zero in Eq. (25). While the $F_{\pi \gamma}\left(Q^{2}\right)$ is sensitive to the shape of the pion DA, the doubly virtual TFF $F_{\pi \gamma^{*}}\left(Q_{1}^{2}, Q_{2}^{2}\right)$ when $Q_{1}^{2} \neq 0$ and $Q_{2}^{2} \neq 0$ is much less sensitive to the end point behavior of the pion DA, since the HSA in Eq. (26) is well behaved at the end points, $x=\{0,1\}$. It can also be noted that, for the kinematic region satisfying $Q_{1}^{2}=Q_{2}^{2}$, the HSA becomes independent of $x$ and thus $Q_{1}^{2} F_{\pi \gamma^{*}}\left(Q_{1}^{2}, Q_{1}^{2}\right)$ only depends on the normalization of the pion DA, which is scale and model independent. One then has

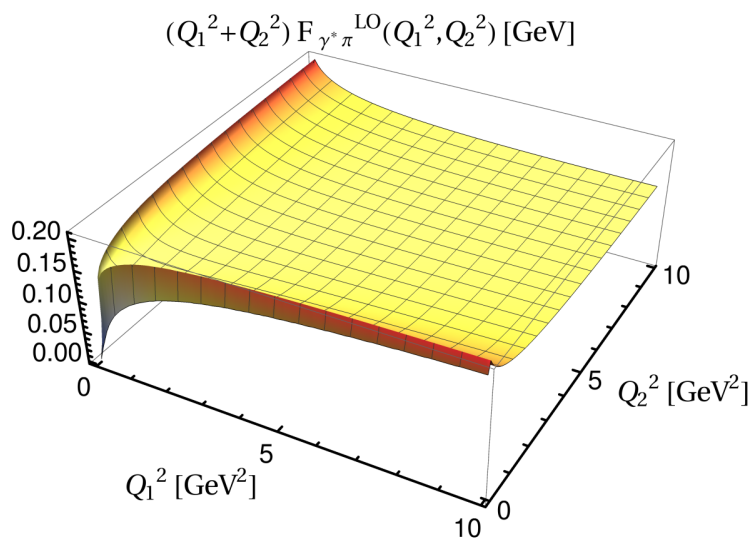

$Q^{2} F_{\pi \gamma^{*}}\left(Q^{2}, Q^{2}\right) \rightarrow \frac{\sqrt{2}}{3} f_{\pi}$. For this transition to two off shell photons, the PQCD expression of $T_{\mathrm{H}}^{\gamma^{*} \gamma^{*} \rightarrow \pi^{0}}$ at NLO can be found in Ref. [29].

The numerical results for $F_{\pi \gamma^{*}}\left(Q_{1}^{2}, Q_{2}^{2}\right)$ within the BLFQNJL model calculated at LO and NLO for selected $\left(Q_{1}^{2}, Q_{2}^{2}\right)$ values are given in Table III. We compare our results with results from the light-front quark model (LFQM) and with results from LO and NLO PQCD [54]. We find that our predictions are close to these results as summarized in Table III. On the other hand, our predictions are very different from the VMD model [51,54]:

$$
F_{\pi \gamma^{*}}^{\mathrm{VMD}}\left(Q_{1}^{2}, Q_{2}^{2}\right)=\frac{F_{\pi \gamma}(0,0)}{\left(1+Q_{1}^{2} / \Lambda_{\rho}^{2}\right)\left(1+Q_{2}^{2} / \Lambda_{\rho}^{2}\right)},
$$

where $\Lambda_{\rho}=775 \mathrm{MeV}$ corresponding to the $\rho$ pole, and $F_{\pi \gamma}(0,0)=0.272(3) \mathrm{GeV}^{-1}$ [102]. The different behavior between our BLFQ-NJL model result and the VMD model prediction can be attributed to the fact that the TFF in the BLFQ-NJL model exhibits $F_{\pi \gamma^{*}}\left(Q_{1}^{2}, Q_{2}^{2}\right) \sim 1 /\left(Q_{1}^{2}+Q_{2}^{2}\right)$ when $\left(Q_{1}^{2}, Q_{2}^{2}\right) \rightarrow \infty$, which is consistent with the PQCD

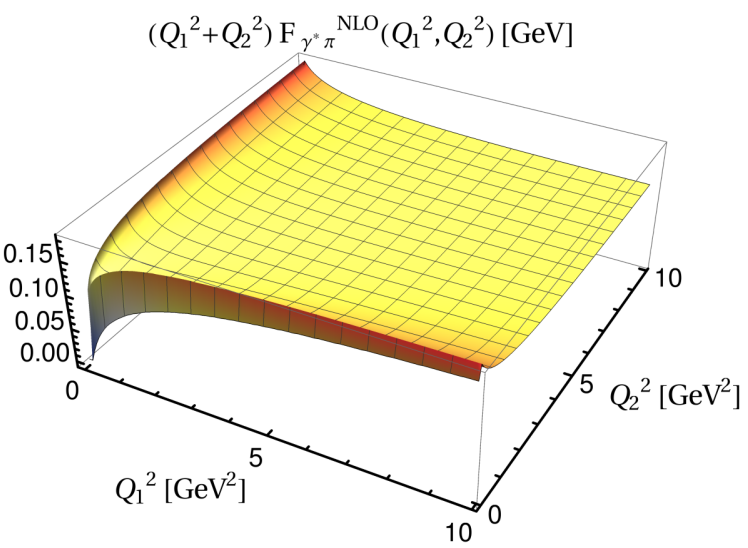

FIG. 5. The three-dimensional plots for the $\pi^{0} \rightarrow \gamma^{*} \gamma^{*}$ transition form factor obtained from Eq. (25) with BLFQ basis truncation $N_{\max }=8$ and $L_{\max }=32$. Left and right panels represent the results with LO and NLO hard scattering amplitude, respectively. Note the difference in the scales on the vertical axes. The effect of the scale evolution in the DA is considered following Eq. (11). 
prediction [1,29], while the TFF in the VMD model behaves as $F_{\pi \gamma^{*}}^{\mathrm{VMD}}\left(Q_{1}^{2}, Q_{2}^{2}\right) \sim 1 /\left(Q_{1}^{2} Q_{2}^{2}\right)$. The consistency between our BLFQ-NJL result and PQCD is expected as the factorization formula in Eq. (25) is applied in our method. Interestingly, for the singly virtual TFF, i.e., for $Q_{1}^{2}=0$ or $Q_{2}^{2}=0$, both the models show the expected scaling behavior $F_{\pi \gamma^{*}}\left(Q^{2}, 0\right) \sim 1 / Q^{2}$ at the large $Q^{2}$ regime.

We show the three-dimensional plots for $\left(Q_{1}^{2}+\right.$ $\left.Q_{2}^{2}\right) F_{\pi \gamma^{*}}\left(Q_{1}^{2}, Q_{2}^{2}\right)$ calculated at LO and NLO in Fig. 5. The qualitative behavior is found to be consistent with the LFQM result [54]. As can be seen from the figure, our BLFQ-NJL model results also show the same scaling behavior as predicted by PQCD $[1,29]$.

\section{SUMMARY}

We have evaluated the valence-quark distribution amplitude from the LFWFs of the pion in the framework of the BLFQ. Our result is based on the wave functions as the eigenfunctions of an effective Hamiltonian which includes the confinement potentials and the color-singlet NambuJona-Lasinio interactions. The meson DA then evolves according to the ERBL evolution equation from PQCD. We have analyzed the QCD evolution of our pion DA and found that it agrees well with the FNAL-E-791 data. At the scale of $10 \mathrm{GeV}^{2}$, our DA is close to the asymptotic QCD prediction.

The nonvanishing moments of the DA in BLFQ framework have been found to be consistent with various theoretical predictions. We have also investigated the sensitivity of the pion DA, decay constants, and the radiative decay width to the BLFQ basis size and found good convergence towards the experimental data with increasing longitudinal basis size.

We have calculated the singly and doubly virtual pionphoton TFFs for $\pi^{0} \rightarrow \gamma^{*} \gamma$ and $\pi^{0} \rightarrow \gamma^{*} \gamma^{*}$ transitions using the DA with the hard-scattering formalism. We have taken into account the LO evolution effects of the pion DA and NLO corrections to the HSA for the calculations at finite $Q^{2}$. We have found that our prediction for $Q^{2} F_{\pi \gamma}\left(Q^{2}\right)$ agrees reasonably well with the Belle Collaboration data, but it disagrees with the rapid growth of the large $Q^{2}$ pionphoton TFF data reported by the BABAR Collaboration. In the meantime, we have observed that the doubly virtual pion-photon TFF in the BLFQ-NJL model is consistent with the LFQM and the PQCD predictions. Our BLFQNJL model result for the doubly virtual TFF manifests as $F_{\pi \gamma^{*}}\left(Q_{1}^{2}, Q_{2}^{2}\right) \sim 1 /\left(Q_{1}^{2}+Q_{2}^{2}\right)$ when $\left(Q_{1}^{2}, Q_{2}^{2}\right) \rightarrow \infty$, which agrees with the $\mathrm{PQCD}$ prediction.

\section{ACKNOWLEDGMENTS}

C. M. is supported by new faculty start up funding by the Institute of Modern Physics, Chinese Academy of Sciences, Grant No. E129952YR0. C. M. and S. N. thank the Chinese Academy of Sciences Presidents International Fellowship Initiative for the support via Grants No. 2021PM0023 and No. 2021PM0021, respectively. X.Z. is supported by new faculty startup funding by the Institute of Modern Physics, Chinese Academy of Sciences, by Key Research Program of Frontier Sciences, Chinese Academy of Sciences, Grant No. ZDB-SLY-7020, by the Natural Science Foundation of Gansu Province, China, Grant No. 20JR10RA067 and by the Strategic Priority Research Program of the Chinese Academy of Sciences, Grant No. XDB34000000. S. J. is supported by U.S. Department of Energy, Office of Science, Office of Nuclear Physics, Contract No. DEAC02-06CH11357. J.P. V. is supported in part by the Department of Energy under Grants No. DE-FG0287ER40371, and No. DE-SC0018223 (SciDAC4/ NUCLEI).
[1] G. P. Lepage and S. J. Brodsky, Phys. Rev. D 22, 2157 (1980).

[2] V. L. Chernyak and A. R. Zhitnitsky, Phys. Rep. 112, 173 (1984).

[3] S. J. Brodsky and G. P. Lepage, Adv. Ser. Dir. High Energy Phys. 5, 93 (1989).

[4] J. C. Collins, L. Frankfurt, and M. Strikman, Phys. Rev. D 56, 2982 (1997).

[5] P. J. Sutton, A. D. Martin, R. G. Roberts, and W. J. Stirling, Phys. Rev. D 45, 2349 (1992).

[6] K. Wijesooriya, P. E. Reimer, and R. J. Holt, Phys. Rev. C 72, 065203 (2005).

[7] M. Gluck, E. Reya, and I. Schienbein, Eur. Phys. J. C 10, 313 (1999).
[8] M. Aicher, A. Schafer, and W. Vogelsang, Phys. Rev. Lett. 105, 252003 (2010).

[9] A. V. Efremov and A. V. Radyushkin, Phys. Lett. 94B, 245 (1980).

[10] M. V. Polyakov, JETP Lett. 90, 228 (2009).

[11] S. Noguera and V. Vento, Eur. Phys. J. A 46, 197 (2010).

[12] C. Terschlusen and S. Leupold, Phys. Lett. B 691, 191 (2010).

[13] V. Y. Petrov, M. V. Polyakov, R. Ruskov, C. Weiss, and K. Goeke, Phys. Rev. D 59, 114018 (1999).

[14] F. Jegerlehner and A. Nyffeler, Phys. Rep. 477, 1 (2009).

[15] A. Nyffeler, Phys. Rev. D 94, 053006 (2016).

[16] A. Gérardin, H. B. Meyer, and A. Nyffeler, Phys. Rev. D 94, 074507 (2016). 
[17] T. Aoyama, N. Asmussen, M. Benayoun, J. Bijnens, T. Blum, M. Bruno, I. Caprini, C. M. Carloni Calame, M. Cè, and G. Colangelo et al., Phys. Rep. 887, 1 (2020).

[18] M. Hoferichter, B. L. Hoid, B. Kubis, S. Leupold, and S. P. Schneider, Phys. Rev. Lett. 121, 112002 (2018).

[19] M. Hoferichter, B. L. Hoid, B. Kubis, S. Leupold, and S. P. Schneider, J. High Energy Phys. 10 (2018) 141.

[20] A. Gérardin, H. B. Meyer, and A. Nyffeler, Phys. Rev. D 100, 034520 (2019).

[21] M. Hoferichter, B. L. Hoid, B. Kubis, and J. Lüdtke, arXiv: 2105.04563.

[22] T. Husek and S. Leupold, Eur. Phys. J. C 75, 586 (2015).

[23] H. J. Behrend, L. Criegee, J. H. Field, G. Franke, H. Jung, J. Meyer, O. Podobrin, V. Schroder, G. G. Winter, P. J. Bussey et al. (CELLO Collaboration), Z. Phys. C 49, 401 (1991).

[24] J. Gronberg, T. S. Hill, Robert K. Kutschke, D. J. Lange, Scott R. Menary, R. J. Morrison, H. N. Nelson, T. K. Nelson, C. Qiao, J. D. Richman et al. (CLEO Collaboration), Phys. Rev. D 57, 33 (1998).

[25] A. Denig (BESIII Collaboration), Nucl. Part. Phys. Proc. 260, 79 (2015).

[26] S. Uehara, Y. Watanabe, H. Nakazawa, I. Adachi, H. Aihara, D. M. Asner, T. Aushev, A. M. Bakich, K. Belous, V. Bhardwaj et al. (Belle Collaboration), Phys. Rev. D 86, 092007 (2012).

[27] B. Aubert, Y. Karyotakis, J. P. Lees, V. Poireau, E. Prencipe, X. Prudent, V. Tisserand, J. G. Tico, E. Grauges, M. Martinelli et al. (BABAR Collaboration), Phys. Rev. D 80, 052002 (2009).

[28] P. del Amo Sanchez, J. P. Lees, V. Poireau, E. Prencipe, V. Tisserand, J. G. Tico, E. Grauges, M. Martinelli, D. A. Milanes, A. Palano et al. (BABAR Collaboration), Phys. Rev. D 84, 052001 (2011).

[29] E. Braaten, Phys. Rev. D 28, 524 (1983).

[30] S. V. Mikhailov and N. G. Stefanis, Nucl. Phys. B821, 291 (2009).

[31] A. V. Radyushkin, Phys. Rev. D 80, 094009 (2009).

[32] A. E. Dorokhov and E. A. Kuraev, Phys. Rev. D 88, 014038 (2013).

[33] X. G. Wu and T. Huang, Phys. Rev. D 82, 034024 (2010).

[34] P. Kroll, Eur. Phys. J. C 71, 1623 (2011).

[35] S. S. Agaev, V. M. Braun, N. Offen, and F. A. Porkert, Phys. Rev. D 86, 077504 (2012).

[36] H. L. L. Roberts, C. D. Roberts, A. Bashir, L. X. GutierrezGuerrero, and P.C. Tandy, Phys. Rev. C 82, 065202 (2010).

[37] S. J. Brodsky, F. G. Cao, and G. F. de Teramond, Phys. Rev. D 84, 075012 (2011).

[38] N. G. Stefanis, A. P. Bakulev, S. V. Mikhailov, and A. V. Pimikov, Phys. Rev. D 87, 094025 (2013).

[39] W. Lucha and D. Melikhov, J. Phys. G 39, 045003 (2012).

[40] J. P. B. C. de Melo, B. El-Bennich, and T. Frederico, FewBody Syst. 55, 373 (2014).

[41] S. S. Agaev, V. M. Braun, N. Offen, F. A. Porkert, and A. Schäfer, Phys. Rev. D 90, 074019 (2014).

[42] H. M. Choi, H. Y. Ryu, and C. R. Ji, Phys. Rev. D 96, 056008 (2017).

[43] H. M. Choi and C. R. Ji, Phys. Rev. D 59, 074015 (1999).

[44] H. M. Choi and C. R. Ji, Phys. Rev. D 75, 034019 (2007).
[45] N. G. Stefanis, Phys. Rev. D 102, 034022 (2020).

[46] M. Ahmady, C. Mondal, and R. Sandapen, Phys. Rev. D 98, 034010 (2018).

[47] T. Zhong, Z. H. Zhu, H. B. Fu, X. G. Wu, and T. Huang, Phys. Rev. D 104, 016021 (2021).

[48] J. Gao, T. Huber, Y. Ji, and Y. M. Wang, arXiv:2106 .01390 .

[49] M. Ding, K. Raya, A. Bashir, D. Binosi, L. Chang, M. Chen, and C. D. Roberts, Phys. Rev. D 99, 014014 (2019).

[50] K. Raya, M. Ding, A. Bashir, L. Chang, and C. D. Roberts, Phys. Rev. D 95, 074014 (2017).

[51] J. P. Lees, V. Poireau, V. Tisserand, E. Grauges, A. Palano, G. Eigen, D. N. Brown, Yu. G. Kolomensky, M. Fritsch, H. Koch et al. (BABAR Collaboration), Phys. Rev. D 98, 112002 (2018).

[52] E. Weil, G. Eichmann, C.S. Fischer, and R. Williams, Phys. Rev. D 96, 014021 (2017).

[53] P. Bickert and S. Scherer, Phys. Rev. D 102, 074019 (2020).

[54] H. M. Choi, H. Y. Ryu, and C. R. Ji, Phys. Rev. D 99, 076012 (2019).

[55] S. J. Brodsky, F. G. Cao, and G. F. de Teramond, Phys. Rev. D 84, 033001 (2011).

[56] A. Stoffers and I. Zahed, Phys. Rev. C 84, 025202 (2011).

[57] H. W. Lin and S. D. Cohen, Proc. Sci. ConfinementX (2012) 113 [arXiv:1302.0874].

[58] X. Feng, S. Aoki, H. Fukaya, S. Hashimoto, T. Kaneko, J. I. Noaki, and E. Shintani, Phys. Rev. Lett. 109, 182001 (2012).

[59] J. J. Dudek and R. G. Edwards, Phys. Rev. Lett. 97, 172001 (2006).

[60] T. Chen, Y. Chen, M. Gong, Yu-H. Lei, N. Li, C. Liu, Yu.-B. Liu, Z. Liu, J. P. Ma, W. F. Qiu et al. (CLQCD Collaboration), Eur. Phys. J. C 76, 358 (2016).

[61] J. P. Vary, H. Honkanen, J. Li, P. Maris, S. J. Brodsky, A. Harindranath, G. F. de Teramond, P. Sternberg, E. G. Ng, and C. Yang, Phys. Rev. C 81, 035205 (2010).

[62] X. Zhao, H. Honkanen, P. Maris, J. P. Vary, and S. J. Brodsky, Phys. Lett. B 737, 65 (2014).

[63] P. Wiecki, Y. Li, X. Zhao, P. Maris, and J. P. Vary, Phys. Rev. D 91, 105009 (2015).

[64] Y. Li and J. P. Vary, arXiv:2103.09993.

[65] Y. Li, P. Maris, X. Zhao, and J. P. Vary, Phys. Lett. B 758, 118 (2016).

[66] Y. Li, P. Maris, and J. P. Vary, Phys. Rev. D 96, 016022 (2017).

[67] S. Jia and J. P. Vary, Phys. Rev. C 99, 035206 (2019).

[68] J. Lan, C. Mondal, S. Jia, X. Zhao, and J. P. Vary, Phys. Rev. Lett. 122, 172001 (2019).

[69] J. Lan, C. Mondal, S. Jia, X. Zhao, and J. P. Vary, Phys. Rev. D 101, 034024 (2020).

[70] L. Adhikari, C. Mondal, S. Nair, S. Xu, S. Jia, X. Zhao, and J. P. Vary, arXiv:2110.05048.

[71] S. Tang, Y. Li, P. Maris, and J. P. Vary, Phys. Rev. D 98, 114038 (2018).

[72] S. Tang, Y. Li, P. Maris, and J. P. Vary, Eur. Phys. J. C 80, 522 (2020).

[73] J. Lan, C. Mondal, M. Li, Y. Li, S. Tang, X. Zhao, and J. P. Vary, Phys. Rev. D 102, 014020 (2020). 
[74] W. Qian, S. Jia, Y. Li, and J. P. Vary, Phys. Rev. C 102, 055207 (2020).

[75] C. Mondal, S. Xu, J. Lan, X. Zhao, Y. Li, D. Chakrabarti, and J. P. Vary, Phys. Rev. D 102, 016008 (2020).

[76] S. Xu, C. Mondal, J. Lan, X. Zhao, Y. Li, and J. P. Vary, arXiv:2108.03909.

[77] J. Lan, K. Fu, C. Mondal, X. Zhao, and J. P. Vary, arXiv:2106.04954.

[78] S. J. Brodsky, G. F. de Teramond, H. G. Dosch, and J. Erlich, Phys. Rep. 584, 1 (2015).

[79] S. Klimt, M. F. M. Lutz, U. Vogl, and W. Weise, Nucl. Phys. A516, 429 (1990).

[80] T. Shigetani, K. Suzuki, and H. Toki, Phys. Lett. B 308, 383 (1993).

[81] U. Vogl, M. F. M. Lutz, S. Klimt, and W. Weise, Nucl. Phys. A516, 469 (1990).

[82] U. Vogl and W. Weise, Prog. Part. Nucl. Phys. 27, 195 (1991).

[83] S. P. Klevansky, Rev. Mod. Phys. 64, 649 (1992).

[84] S. J. Brodsky, H. C. Pauli, and S. S. Pinsky, Phys. Rep. 301, 299 (1998).

[85] A. V. Efremov and A. V. Radyushkin, Theor. Math. Phys. 42, 97 (1980).

[86] E. R. Arriola and W. Broniowski, Phys. Rev. D 66, 094016 (2002).

[87] E. M. Aitala, S. Amato, J. C. Anjos, J. A. Appel, D. Ashery, S. Banerjee, I. Bediaga, G. Blaylock, S. B. Bracker, P. R. Burchat et al. (FNAL-E-791 Collaboration), Phys. Rev. Lett. 86, 4768 (2001).

[88] J. S. Conway, C. E. Adolphsen, J. P. Alexander, K. J. Anderson, J. G. Heinrich, J. E. Pilcher, A. Possoz, E. I. Rosenberg, C. Biino, and J. F. Greenhalgh et al., Phys. Rev. D 39, 92 (1989).

[89] S. J. Brodsky and G. F. de Teramond, Phys. Rev. D 77, 056007 (2008).

[90] N. G. Stefanis, Phys. Lett. B 738, 483 (2014).

[91] P. Ball and R. Zwicky, Phys. Rev. D 71, 014015 (2005).

[92] S. I. Nam, H. C. Kim, A. Hosaka, and M. M. Musakhanov, Phys. Rev. D 74, 014019 (2006).

[93] A. P. Bakulev, S. V. Mikhailov, and N. G. Stefanis, Phys. Lett. B 508, 279 (2001); Phys. Lett. B 590, 309(E) (2004).

[94] V. M. Braun, S. Collins, M. Göckeler, P. Pérez-Rubio, A. Schäfer, R. W. Schiel, and A. Sternbeck, Phys. Rev. D 92 , 014504 (2015).

[95] G. S. Bali, V. M. Braun, M. Göckeler, M. Gruber, F. Hutzler, P. Korcyl, B. Lang, A. Schäfer (RQCD Collaboration), Phys. Lett. B 774, 91 (2017).

[96] G. S. Bali, V. M. Braun, S. Bürger, M. Göckeler, M. Gruber, F. Hutzler, P. Korcyl, A. Schäfer, A. Sternbeck, P. Wein (RQCD Collaboration), J. High Energy Phys. 08 (2019) 065.

[97] R. Zhang, C. Honkala, H. W. Lin, and J. W. Chen, Phys. Rev. D 102, 094519 (2020).
[98] S. S. Agaev, Phys. Rev. D 72, 114010 (2005); Phys. Rev. D 73, 059902(E) (2006).

[99] L. Chang, I. C. Cloet, J. J. Cobos-Martinez, C. D. Roberts, S. M. Schmidt, and P. C. Tandy, Phys. Rev. Lett. 110, 132001 (2013).

[100] R. Arthur, P. A. Boyle, D. Brommel, M. A. Donnellan, J. M. Flynn, A. Juttner, T. D. Rae, and C. T. C. Sachrajda, Phys. Rev. D 83, 074505 (2011).

[101] V. M. Braun, M. Gockeler, R. Horsley, H. Perlt, D. Pleiter, P. E. L. Rakow, G. Schierholz, A. Schiller, W. Schroers, H. Stuben et al., Phys. Rev. D 74, 074501 (2006).

[102] M. Tanabashi, K. Hagiwara, K. Hikasa, K. Nakamura, Y. Sumino, F. Takahashi, J. Tanaka, K. Agashe, G. Aielli, C. Amsler et al. (Particle Data Group), Phys. Rev. D 98, 030001 (2018).

[103] S. L. Adler, Phys. Rev. 177, 2426 (1969).

[104] J. S. Bell and R. Jackiw, Nuovo Cimento A 60, 47 (1969).

[105] S. J. Brodsky and G. P. Lepage, Phys. Rev. D 24, 1808 (1981).

[106] F. del Aguila and M. K. Chase, Nucl. Phys. B193, 517 (1981).

[107] E. P. Kadantseva, S. V. Mikhailov, and A. V. Radyushkin, Yad. Fiz. 44, 507 (1986).

[108] B. Melic, B. Nizic, and K. Passek, Phys. Rev. D 65, 053020 (2002).

[109] B. Melic, D. Mueller, and K. Passek-Kumericki, Phys. Rev. D 68, 014013 (2003).

[110] J. Gronberg, T. S. Hill, R. K. Kutschke, D. J. Lange, S. R. Menary, R. J. Morrison, H. N. Nelson, T. K. Nelson, C. Qiao, J. D. Richman et al. (CLEO Collaboration), Phys. Rev. D 57, 33 (1998).

[111] H. J. Behrend, L. Criegee, J. H. Field, G. Franke, H. Jung, J. Meyer, O. Podobrin, V. Schroder, G. G. Winter, P. J. Bussey et al. (CELLO Collaboration), Z. Phys. C 49, 401 (1991).

[112] S. V. Mikhailov and N. G. Stefanis, Mod. Phys. Lett. A 24, 2858 (2009).

[113] A. P. Bakulev, S. V. Mikhailov, A. V. Pimikov, and N. G. Stefanis, Phys. Rev. D 84, 034014 (2011).

[114] X. G. Wu and T. Huang, Phys. Rev. D 84, 074011 (2011).

[115] A. E. Dorokhov, Phys. Part. Nucl. Lett. 7, 229 (2010).

[116] H. N. Li and S. Mishima, Phys. Rev. D 80, 074024 (2009).

[117] E. R. Arriola and W. Broniowski, Phys. Rev. D 81, 094021 (2010).

[118] M. Gorchtein, P. Guo, and A. P. Szczepaniak, Phys. Rev. C 86, 015205 (2012).

[119] T. N. Pham and X. Y. Pham, Int. J. Mod. Phys. A 26, 4125 (2011).

[120] A. E. Dorokhov, JETP Lett. 92, 707 (2010).

[121] S. S. Agaev, V. M. Braun, N. Offen, and F. A. Porkert, Phys. Rev. D 83, 054020 (2011).

[122] P. Kotko and M. Praszalowicz, Phys. Rev. D 80, 074002 (2009). 